\title{
Diameter of the Inferior Alveolar Canal - A Comparative CT and Macroscopic Study of Sudanese Cadaveric Mandibles
}

\author{
Mashail Mahmoud Hamid ${ }^{1}$, Ahmed Mohamed Suliman² \\ ${ }^{1}$ Department of Oral and Maxillofacial Surgery, College of Dentistry, King Khalid \\ University, Abha, Saudi Arabia ${ }^{2}$ Department of Oral and Maxillofacial Surgery, \\ University of Khartoum, Sudan.
}

\section{ABSTRACT}

\section{BACKGROUND}

CT scan is a reliable diagnostic method of head and neck pathology. In patients with oral cancer, it is of great value in detecting bone invasion, as well as perineural spread. The latter usually results in widening of the bony canals and foramina. This study aimed to validate the use of CT scan for assessment of inferior alveolar canal (IAC) dimensions, measure the dimensions of the inferior alveolar canal at different sites in normal cadaveric mandibles and determine the accuracy of CT scan in measuring inferior alveolar canal dimensions.

\section{METHODS}

In this observational study, five complete human cadaveric mandibles were marked at six different positions from the distal border of the mental foramina. The mandibles were CT scanned in the coronal plane at the marked positions and the inner width and height of the canal were measured. Afterwards, the specimens were sectioned longitudinally at matching locations of CT cuts marked positioned and canal dimensions were measured macroscopically using a digital calliper. The two sets of measurements were then compared. Right and left sides measurements were also compared.

\section{RESULTS}

Pearson correlation coefficient showed moderate linear correlation for measured variables. Regarding the height of the canal, the findings showed no significant difference between the two methods ( $p>0.05)$. However, for the width, there was significant statistical difference between CT measurements and the manual ones ( $p<$ $0.05)$. A statistically significant difference was also found in the dimensions of the canal between the right and left sides $(\mathrm{p}<0.05)$

\section{CONCLUSIONS}

Measuring the inferior alveolar canal dimension using CT scan is not absolutely accurate.

\section{KEY WORDS}

Computed Tomography (CT), Inferior Alveolar Canal (IAC), Diameter
Corresponding Author:

Dr. Mashail Mahmoud Hamid,

Department of Oral and Maxillofacial

Surgery, College of Dentistry, King Khalid University, Abha, Saudi Arabia and Faculty of Dentistry, University of Khartoum, Sudan.

E-mail: mashailmahmoud@gmail.com

\section{DOI: $10.14260 /$ jemds/2021/77}

How to Cite This Article:

Hamid MM, Suliman AM. Diameter of the inferior alveolar canal- a comparative CT and macroscopic study of sudanese cadaveric mandibles. J Evolution Med Dent Sci 2021;10(06):342-346, DOI: 10.14260/jemds/2021/77

Submission 25-07-2020,

Peer Review 11-12-2020,

Acceptance 17-12-2020,

Published 08-02-2021.

Copyright (C) 2021 Mashail Mahmoud Hamid et al. This is an open access article distributed under Creative Commons Attribution License [Attribution 4.0 International (CC BY 4.0)] 


\section{BACKGROUND}

Inferior alveolar canal is the bony canal within the mandible that contains the inferior alveolar nerve and associated vascular structures are located between the mandibular foramen and mental foramen. Its cross-sectional shape has been described as oval and round. The average diameter of the mandibular canal has been reported to be $3.4 \mathrm{~mm}$. The inferior alveolar nerve passes through the infratemporal fossa and ends in the mandible where it usually divides into incisive and mental branches. ${ }^{1}$

IAC is regarded as one of the most significant anatomical structures in the head region. Thus, knowledge of its path and position is essential in reducing the risk of nerve damage during implant placement, mandibular nerve reposition procedures, ramus, and symphysis bone graft surgery, sagittal split osteotomies, and monocortical plating. The canal diameter could also be affected by a variety of diseases of bone, either by a decrease in size or by an increase in diameter as in the case of osteolytic lesions such as oral malignancies. ${ }^{2-4}$

In the oral and maxillofacial region, tumours tend to spread along the trigeminal and facial nerves. In the mandible, perineural invasion occurs along the mandibular nerve (the 3rd division of the trigeminal nerve) and in particular via the inferior alveolar nerve. CT scans have been widely used to detect such invasion in addition to soft issued involvement and bone metastases. ${ }^{5-6}$

Globally, the incidence of oral cancers is increasing, the same is observed in Sudan. ${ }^{7}$ Radical removal of cancerous tissues offers the best treatment of the disease. The latter can only be achieved if it is contemplated with good safety margins. An important issue of concern in malignancy is metastasis. In the mandible, the inferior alveolar canal acts like a subway facilitating the spread of malignancy along its course. Detection of any changes in its diameter in a cancer patient should be taken into consideration when planning resection of borders. At present, in these cases, CT scan offers the best imaging modality for detecting any changes in the canal diameter.

The knowledge of the actual diameter of the canal helps in the detection of any abnormal changes in its size. This study is a comparative study between the macroscopic and the CT scan measurements of the canal in cadaveric mandible. It is hoped that such work shall help in establishing a standard reference for interpretation of pathological changes involving the canal.

\section{METHODS}

This observational study was approved by the Ethics Committee and Postgraduate Medical and Health Science Board, Faculty of Dentistry, University of Khartoum.

The specimens in this study were five complete adult human cadaveric mandibles obtained from the Department of Anatomy, Faculty of Medicine, University of Khartoum. Mandibles with any abnormality or not of a complete nature were excluded from the study. Five dentate complete mandibles were selected and preserved in formalin $10 \%$ medium. Each mandible was marked at six positions from the distal border of the mental foramen and in a posterior direction at intervals of $10 \mathrm{~mm}$ on both right and left sides on each mandible at the six sites namely $\mathrm{X} 1, \mathrm{X} 2, \mathrm{X} 3, \mathrm{X} 4, \mathrm{X} 5$, and X6, parallel lines were drawn. Gutta Percha (GP) points, known as markers, were attached to the buccal aspect of dry mandibles on $\mathrm{X} 1, \mathrm{X} 2, \mathrm{X} 3, \mathrm{X} 4, \mathrm{X} 5$, and $\mathrm{X} 6$. The mandibles were scanned at the marked positions with $5 \mathrm{~mm}$ collimation and a table speed of $5 \mathrm{~mm} / \mathrm{s}$ using helical (spiral) CT with a "G.D dual scanner made by Siemens Medical Systems, Erlangen, Germany".

Inner width and height of the canal cross-section at the six positions were recorded (Figure 1). The mandibles were then sectioned using the electrical bone saw at the marked positions. The same measurements were taken again macroscopically using an electronic digital calliper and recorded.

The collected data was analysed using STATA software version 10 . The variables being studied were the height and width of the inferior alveolar canal measured via CT scan and again measured macroscopically. The statistical analysis describing the distribution of the measures in terms of minimum, maximum, mean, Standard Deviation (SD), and Standard Error of The Mean (SEM).

The statistical analysis focused on comparing the radiological mean height and width of the canal versus the macroscopic height and width. Differences were assessed using the paired t-test for comparison of means at a level of significance of 0.05 . A simple linear regression model was used, and the correlation coefficient was calculated.

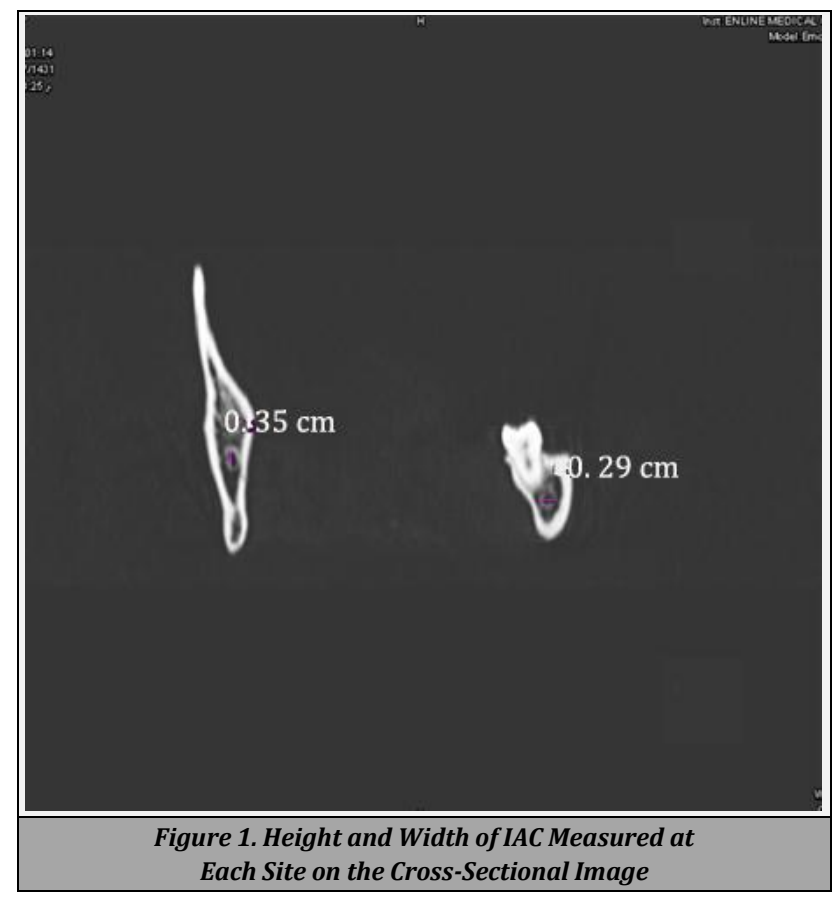

\section{RESULTS}

Five adult Sudanese cadaveric mandibles were included in this study, in which the diameter of the inferior alveolar canal was measured in both vertical and horizontal planes. In all mandibles, the canal appeared as a single structure. It was visible in 57 sites out of 60 sites. i.e., with $95 \%$ visibility.

The mean height of the canal measured macroscopically was $0.35 \mathrm{~cm}$, and $0.34 \mathrm{~cm}$ on CT scans. Whereas, the mean width of the canal measured macroscopically was $0.29 \mathrm{~cm}$, and 
$0.27 \mathrm{~cm}$ on CT scans. There was no significant difference in height values between the two methods ( $p$-value $>0.05$ ). On the contrary, width measurements showed a difference which was statistically significant between the two methods ( $p$-value $=0.0035$ ). Furthermore, a positive and moderate correlation was observed between radiographic and macroscopic measurements. (Figures $2 \& 3$ ). The correlation regarding the height was $59.12 \%$ and $62.18 \%$ for the width of the canal.

Furthermore, a simple linear regression test was performed. The relation of the variables was assumed by the following the equation " $y=\alpha+\beta x$ " in which " $y$ " reflects the variable measured by $\mathrm{CT}$ and " $\mathrm{x}$ " reflects the variable measured manually, the results were:

- For the height: H-R $=0.098+0.685$ H-M (Height Macroscopically).

- For the width: W-R $=0.058+0.734$ W-M (Width Macroscopically).

Right, and left side measurements were further compared. Mean values, SD, and SEM and $95 \%$ Confidence Interval (CI) of height and width measurements are presented in Table 1 . In addition to that, t-test of equality of means between the measurements on the right and left sides was performed and it revealed the following:

- Macroscopically: For the measurement obtained for the height, $\mathrm{P}$ value was 0.0107 while for the width P value was 0.0214. (Statistically significant difference for both).

- Radiologically: P value for height measurement was 0.0288 , (statistically significant difference), while for the width, the $\mathrm{P}$ value was 0.0897 indicating statistically nonsignificant difference.

The values of the means and the standard deviations of canal dimensions at each location are presented in Table 2.

\begin{tabular}{|ccccccccc|}
\hline \multicolumn{1}{|c|}{ Right side } & \multicolumn{2}{c|}{ Left side } & \multicolumn{2}{c|}{ Right side } & \multicolumn{2}{c|}{ Left side } \\
& H-R & H-M & H-R & H-M & W-R & W-M & W-R & W-M \\
Mean & 0.31 & 0.33 & 0.36 & 0.38 & 0.26 & 0.28 & 0.29 & 0.31 \\
SD & 0.05 & 0.05 & 0.08 & 0.07 & 0.05 & 0.04 & 0.06 & 0.05 \\
SEM & 0.01 & 0.01 & 0.01 & 0.01 & 0.01 & 0.00 & 0.01 & 0.01 \\
95\% & $0.29-$ & $0.31-0.350 .33-0.39$ & $0.35-$ & $0.24-$ & $0.26-$ & $0.26-0.31$ & $0.29-$ \\
CI & 0.34 & 0.40 & 0.28 & 0.29 & $0.26-0.33$ \\
\hline \multicolumn{18}{|c|}{ Table 1. The Mean Difference between the Right and Left Sides } \\
for the Height and Width \\
\hline
\end{tabular}

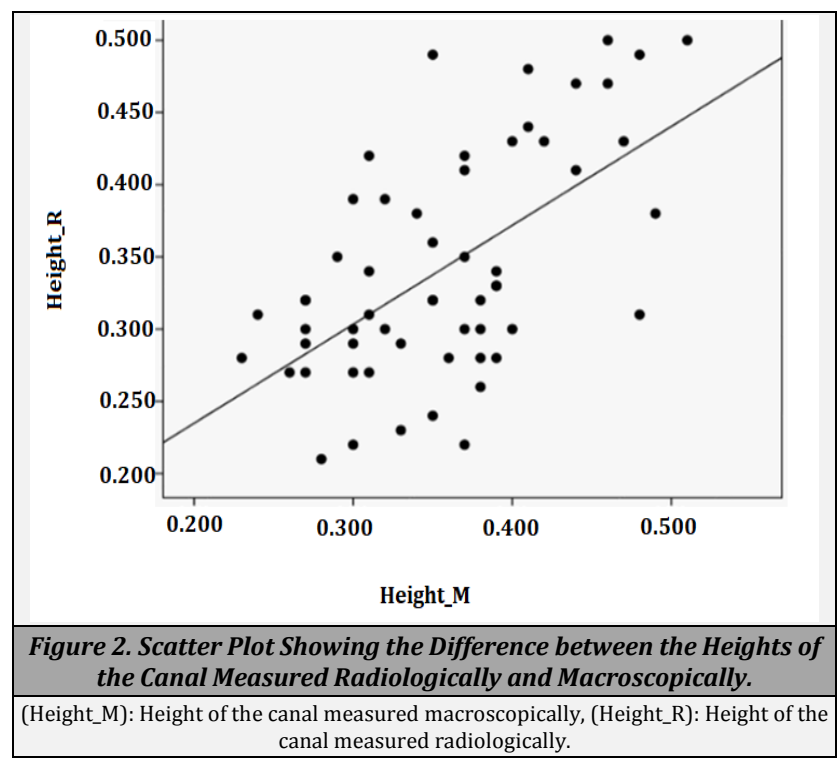

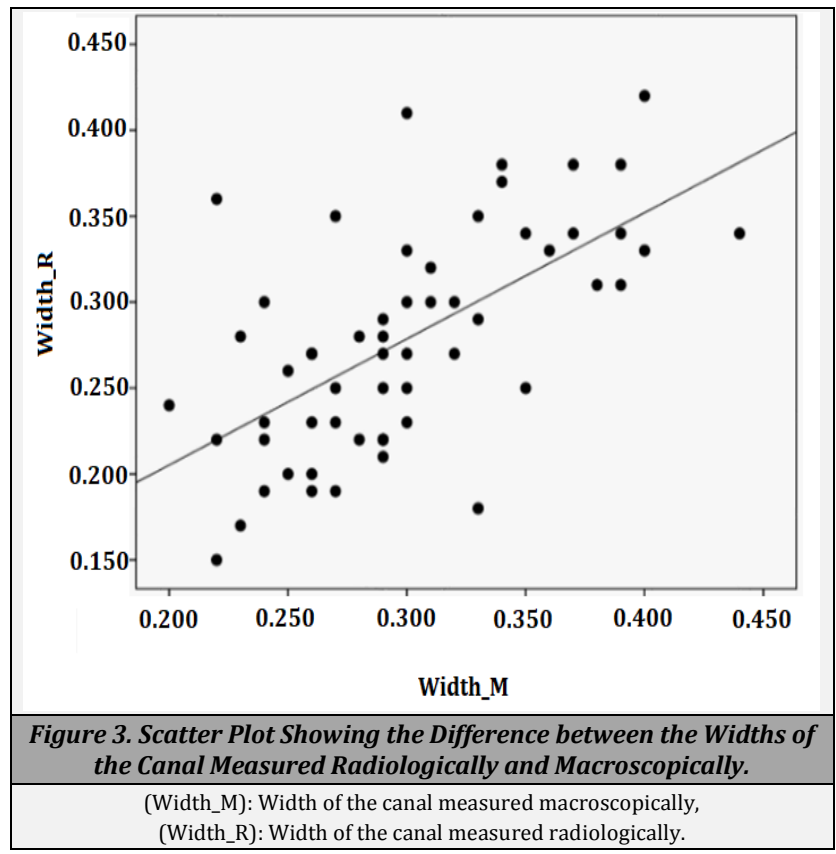

\begin{tabular}{|ccccc|}
\hline $\begin{array}{c}\text { Position } \\
\text { M: Macroscopically } \\
\text { R: Radiographically }\end{array}$ & Height_R & $\begin{array}{c}\text { Height_M } \\
\text { Width_R }\end{array}$ & Width_M \\
X1M & $0.38 \pm 0.09$ & $0.40 \pm 0.1$ & $0.33 \pm 0.06$ & $0.38 \pm 0.06$ \\
X1R & $0.32 \pm 0.07$ & $0.30 \pm 0.04$ & $0.26 \pm 0.02$ & $0.26 \pm 0.03$ \\
X2M & $0.40 \pm 0.10$ & $0.39 \pm 0.06$ & $0.29 \pm 0.08$ & $0.29 \pm 0.06$ \\
X2R & $0.35 \pm 0.09$ & $0.37 \pm 0.06$ & $0.24 \pm 0.06$ & $0.27 \pm 0.02$ \\
X3M & $0.43 \pm 0.08$ & $0.42 \pm 0.07$ & $0.33 \pm 0.03$ & $0.31 \pm 0.04$ \\
X3R & $0.31 \pm 0.03$ & $0.37 \pm 0.08$ & $0.29 \pm 0.05$ & $0.31 \pm 0.05$ \\
X4M & $0.3 \pm 0.07$ & $0.35 \pm 0.06$ & $0.27 \pm 0.1$ & $0.32 \pm 0.06$ \\
X4R & $0.35 \pm 0.04$ & $0.33 \pm 0.03$ & $0.31 \pm 0.07$ & $0.32 \pm 0.06$ \\
X5M & $0.33 \pm 0.1$ & $0.34 \pm 0.07$ & $0.27 \pm 0.05$ & $0.31 \pm 0.05$ \\
X5R & $0.27 \pm 0.04$ & $0.32 \pm 0.04$ & $0.25 \pm 0.02$ & $0.28 \pm 0.05$ \\
X6M & $0.36 \pm 0.08$ & $0.39 \pm 0.07$ & $0.26 \pm 0.07$ & $0.28 \pm 0.05$ \\
X6R & $0.33 \pm 0.06$ & $0.32 \pm 0.07$ & $0.23 \pm 0.05$ & $0.25 \pm 0.04$ \\
\hline \multicolumn{5}{c}{ Table 2. Values of the Mean and the } \\
\hline \multicolumn{5}{c}{ Standard Deviation at Each Location } \\
\hline
\end{tabular}

\section{DISCUSSION}

Knowledge of the anatomy of the inferior alveolar canal and its variations is of great importance to the oral and maxillofacial surgeon. Currently, CT plays a key role and is the most accurate diagnostic tool in oral \& maxillofacial surgery. Nonetheless, it often fails to determine the actual dimensions of the IAC. Therefore, knowledge of the real dimensions of this canal on actual mandibles is of importance for validation and evaluation of the different imaging modalities in use.

Many researchers have studied the anatomy, including location, shape, and course of the inferior alveolar canal with conventional radiography, ${ }^{8-10}$ but few of them have concentrated on studying the size of the canal i.e., its dimensions. The main objective of this study was to evaluate the ability of the CT scan in determining the exact dimensions of the inferior alveolar canal.

\section{Visibility of the Canal}

The current study describes the visibility of the mandibular canal on CT scan. The canal could be observed clearly in $95 \%$ of the total sites studied on the CT scan and with fair visibility 
in most of these cases. However, in some few cases it was difficult to differentiate between the canal and the adjacent bone spaces. The results of the present study were in accordance with another study by Jacobe and colleagues, where the canal was visible in $97 \%$ of the cases. ${ }^{11}$

The visibility of the canal depends on the presence of welldefined cortical borders which results in better contrast between the contour of the canal and the surrounding bone . According to Lindh et al, this explains why CT images taken in coronal plane showed the highest number of clearly visible canals .On the other hand, it also explains why the reconstructed images from CT have got the poorest visualization compared to other methods used in their study. ${ }^{12}$

\section{Accuracy of CT}

The results of CT were slightly underestimated when compared to the manual measurements. On CT scan, the mean height as well as the mean width of the inferior alveolar canal were smaller than those measured manually. The t-test of equality of means was performed with the null hypothesis. The mean height of the canal measured radiographically and the one measured manually was equal at 0.05 level of significance.

Concerning the dimensions of the canal, there was no statistical difference between both modalities of measurements i.e. the CT scan and the direct manual method with regard to the height ( $p$-value was found to be 0.1055 ), On the other hand, there was a significant statistical difference between the width of the canal measured from CT and the width when measured manually ( $\mathrm{p}$-value $=0.0035$ ).

When the two modalities were correlated to each other, there was a positive correlation between them. This correlation found to be moderate, amounting to $59.12 \%$ for the height and to $62.18 \%$ for the width.

Peker et al reported that CT scan was an excellent tool for detection and localization of IAC with a magnification rate of $3.86 \%$ when compared to other images. Moreover, they reported a strong correlation between measurements obtained by CT and the direct manual measurements. ${ }^{13}$

Accuracy in measurements obtained radiologically depends on the degree of cortication of the canal and the integrity of its cortical lining. In addition to that, it is also influenced by the subjectivity and experience of the interpreter. These factors have contributed to the variability between the radiological dimensions and the actual ones and explain why this variation appeared to occur in one variable (the width) but not in the other one (the height).

When using the simple linear regression test to relate the radiological measurements to the actual manual measurements, both width and height showed no significant differences. The H-R (Height Radiologically) and the W-R (Width Radiologically) equalled $0.098+0.685 \mathrm{H}-\mathrm{M}$ and $0.58+$ $0.734 \mathrm{~W}$-M respectively.

\section{Diameter of the IAC on Right and Left Sides}

The t-test of equality of means was performed on our variables. Regarding the measurement obtained macroscopically: for the height, the p-value was 0.0107 while for the width, the p-value was 0.0214. The difference in the values was statistically significant at a confidence level of 0.05 .

These results demonstrated that the dimensions of the canal are not equal on both sides i.e. the canal tends to be asymmetrical in its dimensions. However, with regard to the radiological measurements, for the height, the p-value was 0.028 and the difference was statistically significant, while for the width, the $\mathrm{p}$-value was 0.0897 and the difference was not statistically significant.

\section{Shape and Diameter}

Regarding the shape and diameter of the canal, this study showed that the canals observed were not uniform, and their diameters varied at the different sites. The mean diameter in the vertical plane (the height), when measured radiologically was $0.34 \mathrm{~cm}$; while the mean diameter in the horizontal plane (the width) was $0.27 \mathrm{~cm}$. However, the manual measurement of these dimensions showed that the mean height was $0.35 \mathrm{~cm}$ while the mean width was $0.29 \mathrm{~cm}$. The canal was oval in most of the cases. In the remaining few cases, the canal was round in shape in four different sites. i.e. the vertical and horizontal diameters of the canal were equal, but radiologically this appeared to occur in one site only. Generally, the canal appeared to be wider in its middle part.

These results are close to those obtained by Ikeda and colleagues, who used multiplanar MRI (Magnetic Resonance Imaging) for evaluation of the IAC and found that the canal was oval in shape and its greater diameter averaged $4.1 \mathrm{~mm}$ (sd 0.5 $\mathrm{m}$ ) near the mandibular foramen, while in its middle part, the canal was round in shape with an average diameter of $3.4 \mathrm{~mm}$ (sd $0.5 \mathrm{~mm}$ ). However, in this study, larger values appeared to occur in the middle part of the canal. ${ }^{1}$

In a study by Ylikontiola et al, using ct scan, the diameter of the mandibular canal ranged from $1.2 \mathrm{~mm}$ to $3.0 \mathrm{~mm}$, with a mean of $2.1 \mathrm{~mm}$ but anteriorly, the diameter of the IAC ranged from $1.3 \mathrm{~mm}$ to $2.7 \mathrm{~mm}$, with a mean of $2 \mathrm{~mm} .{ }^{14}$

It is obvious that there are clear differences between our findings and Ylikontioa findings. These variations may be a result of many confounding factors including the experience and capability of the observer or interpreter (personal) or, type and standard of the equipment and / or calibrator used (technical). The observer indeed requires certain experience, skills and knowledge on-base information to enable determining the exact anatomical features of the canal.

\section{Limitations of the Study}

This study has a number of limitations.

1. The sample size was small due to limited supply of autopsy specimens.

2. The study subjects were dry mandibles that had probably shrunk and certainly are different from those of living patients. The IAC of the patient is much more clearly visualized than that of the dry mandible .Thus, the measurement error on the dry mandible could be higher than that on the patient.

3. The collected sample was short of information regarding the age and gender parameters. The availability of this information could have resulted in a more comprehensive study.

4. In the present study, the diameter of the IAC was evaluated by focusing only on six sites at intervals of 10 $\mathrm{mm}$. Further research with closer intervals, might give more reliable results and helps in validating the accuracy of the diameter of the inferior alveolar canal. 


\section{CONCLUSIONS}

Measurements of the inferior alveolar canal obtained from the CT scan were different from measurements of the canal taken macroscopically. A positive and moderate correlation was observed between radiographic and macroscopic measurements. A significant difference in IAC dimensions between the left and right sides exists. Height and width of the IAC canal varied at different sites.

Data sharing statement provided by the authors is available with the full text of this article at jemds.com.

Financial or other competing interests: None.

Disclosure forms provided by the authors are available with the full text of this article at jemds.com.

\section{REFERENCES}

[1] Ikeda K, Ho KC, Nowicki BH, et al. Multiplanar MR and anatomic study of the mandibular canal. AJNR Am J Neuroradiol 1996;17(3):579-84.

[2] Mojover YN, Sahebjamie M, Tirgary F, et al. Enlargement of mandibular canal with tongue paresthesia caused by extranodal B-cell lymphoma: a case report. Oral Oncol 2005;41(5):97-9.

[3] Larheim TA, Westesson PLA. Maxillofacial imaging. Springer-Verlag Berlin Heidelberg 2006:6-7.

[4] Yamada T, Kitagawa Y, Ogasawara T, et al. Enlargement of mandibular canal without hypesthesia caused by extranodal non-Hodgkin's lymphoma: a case report. Oral Surg Oral Med Oral Pathol Oral Radiol Endod 2000;89(3):388-92.
[5] Mukherji SK, Isaacs DL, Creager A, et al. CT detection of mandibular invasion by squamous cell carcinoma of the oral cavity. AJR Am J Roentgenol 2001;177(1):237-43.

[6] Ostuni A. Use of computed tomography in diagnosis,staging and treatment of squamous cell carcinoma of the mandible. Columbia Dental Review 2001;6:22-7.

[7] Salah MA, Mohamed EA. Frequency of oral cancers among cancers in Sudanese patients (2009-2016). J Adv Med Dent Sci Res 2017;5(9):26-35.

[8] Kieser JA, Paulin M, Law B. Intrabony course of the inferior alveolar nerve in the edentulous mandible. Clin Anat 2004;17(2):107-11.

[9] Claeys V, Wackens G. Bifid mandibular canal: literature review and case report. Dentomaxillofac Radiol 2005;34(1):55-8.

[10] Kalabalik F, Aytugar E. Localization of the mandibular canal in a Turkish population: a retrospective cone-beam computed tomography study. J Oral Maxillofac Res 2019;10(2):e2.

[11] Jacobs R, Mraiwa N, Van Steenburghe D, et al. Appearance of the mandibular incisive canal on panoramic radiographs. Surg Radiol Anat 2004;26(4):329-33.

[12] Lindh C, Petersson A, Klinge B. Visualisation of the mandibular canal by different radiographic techniques. Clin Oral Implants Res 1992;3(2):90-7.

[13] Peker I, Alkurt MT, Michcioglu T. The use of 3 different imaging methods for the localization of the mandibular canal in dental implant planning. Int J Oral Maxillofac Implants 2008;23(3):463-70.

[14] Ylikontiola L, Moberg K, Huumonen S, et al. Comparison of three radiographic methods used to locate the mandibular canal in the buccolingual direction before bilateral sagittal split osteotomy. Oral Surg Oral Med Oral Pathol Oral Radiol Endod 2002;93(6):736-42. 